Rev. Chil. Pedlatr. 66 (3); 131-135, 1995

\title{
Broncodilatadores en Síndrome Bronquial Obstructivo del lactante
}

\author{
Mario Calyo G. '; Francisco Marín $\mathrm{H}^{2}$; Carmen Vera M. ${ }^{3}$; Karin Grob B. ${ }^{4}$; \\ Carmen Albornoz V. ${ }^{4}$; Marion Hermann H. ${ }^{5}$, Mario Calvo A. ${ }^{6}$
}

\section{Bronchodilator agents in infants with acute obstructive bronchial syndrome}

\begin{abstract}
Clinica ressanses to four different treatmen regimers given by nebulization to 100 children under age. 24 month w:th ocute obstructive broncinjal synerome which recuired hospitalizalion, were evoluated through a dousle blind prospective study. Twenty rive patients were osignec to eact of 'our groups: group I subjects were given ipratropium H. group II ch ldren receivad sol.ne sclution 10.9\% VoCli, group III purients recelived a combination of fenoterol and iprairspium ifl) and group IV child en were given salbutamo. (S). Tal sco:es were similar at admission in a'l four groups

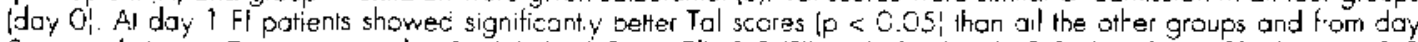

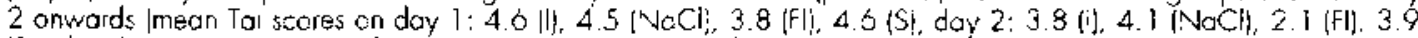
(S); day 3. 3.0 ill. 3.4 i NaCl], 1.6 ifl, 3.2 ist respectively ip < 0.01 i. Average days in hospirol were l: $4.04 \pm$ 29: AaCl: $5.44 \pm 1.01: \mathrm{Fl}: 3.08 \pm 1.28, \mathrm{~S} 5.28 \pm 1.69 ; \mathrm{p}<0.001$ in :ovor of fi. Requirements for additional bronchodi'ator theropy were higher $\mathrm{fc}_{\mathrm{c}} \cdot \mathrm{NaCl}$ treated patients $(\mathrm{p}<0.05$ ) from doy 2 , and lower for $\mathrm{F}$ a asigned infonls from doy $110<0.01$ i. No urdesiable seccncary effects were obseved. in criidren under two years of oge with bronchial obs:rucive syndrome, the best therapeuf. e effect was shewn by combined tenolerol-ipratropium.
\end{abstract}

IKey words: in:ant, ccu'e obstructive brenchial sy-drome. Fenoteral pratropium, salbutamol, ipral:opium.i

El uso de broncodilatadores en los lactantes que sufren una crisis bronquial obstructiva y la elección del medicamento más apropiado continúan siendo materia de controversia, particularmente en los niños de menor edad.

La mezcla fenoterol-ipratropio suministrada por nebulización ha producido, en lactantes con síndrome bronquial obstructivo ( $\mathrm{SBO}$ ), tiene efectos favorables en comparación con la solucion de $\mathrm{NaCl} 0,9 \%^{1}$. En otras experiencias se ha empleado salbutamol, ipratropio o mezclas de un beta 2 adrenérgico con un anticolinêrgico $^{2-6}$.

El estudio cuyos resultados se describen se realizó con el propósito de comparar el efecto

I. Instituto de Pediatría

2. Bioestadístico Instituto de Salud Pública

3. Becado. Instituto de Pediatría, Universidad Austral

4. Servicio Salud Valdivia

5. Enfermera Universitaria. Servicio Salud Valdivia

6. Ayudante alumno. Instituto de Pediatría, Universidad Austral. broncodilatador de salbutamol, ipratropio y fenoterol-ipratropio nebulizados en lactantes con síndrome bronquial obstructivo, utilizando como controles a pacientes que recibieron sólo nebulizaciones de $\mathrm{NaCl} 0,9 \%$.

\section{Material y Método}

Entre los meses de octubre de 1993 a febrero de 1994 se reclutaron para el estudio todos los lactantes adrnitidos por síndrome bronquial obstructivo agudo a la unidad de iactantes del Servicio de Pediatría del Hospital Regional de Valdivia, hasta completar un total de 100 niños. De acuerdo a su orden de ingreso se asignaron 25 vínos a cada uno de los cuatro grupos a los cuales se administro, con un sistema de doble ciego, nebulizaciones de los medicamentos que se indican en cada caso, cada 6 horas, mediante nebulizador Hudson. con flujo de oxígeno de 6 litros por minuto. A la dosis indicada de cada medicamento se agregó solución de $\mathrm{NaCl} 0,9 \%$ para completar un volumen total de $4 \mathrm{ml}$ a nebulizar. Los pacientes asignados al grupo I (l) recibieron bromaro de ipratropio $(0.25 \mathrm{mg} / \mathrm{ml})$ dosis $0,1 \mathrm{ml} \cdot \mathrm{kg} \cdot$ dosis máximo $1 \mathrm{ml}$ por dosis. Los del grupo II $(\mathrm{NaCl})$ fueron tratados con $\mathrm{NaCl} 0,9 \% 0,1 \cdot \mathrm{ml} \mathrm{kg}$. dosis, máximo 1 ml por dosis. A los lactantes del grupo III 
(FI) les fue suministrado bromhidrato de fenoterol 10.5 $\mathrm{mg} / \mathrm{ml})$ y bronuro de ipratropio $(0,25 \mathrm{mg} / \mathrm{ml}) 0,1 \mathrm{ml} \cdot \mathbf{k g}$ * dosis, máximo I ml por dosis. Los niños del grupo IV (S) recibiersn salbutamol ( $5 \mathrm{mg} / \mathrm{ml}) 0.5 \mathrm{ml}$ por dosis.

En todos ellos se registró, al ingreso y en en los días siguientes, cl puntaje de Tal ${ }^{7}$ modificado en Chile (tabla 1). antibisticos. otros broncodilatadores o corticoides agregados al tratumiento basal y las reacciones adversas a los medicamentos (taquicardia, temblor, enrojeciniento facial. vómitos, sequedad de mucosas y excicación sicomotora).

La evolución clínica se califico como desfarorable si, transcurridas 24 horas con el tratamiento experimedtal, se mantuviera o aumentara en los pacientes la calificación Tal Jel ingreso. agregándose a la terapio dos inhalaciones de

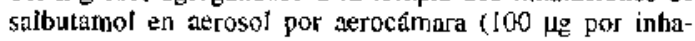
lación) vada 6 horas y. según la seyeridad de los sintomas, hidrocottisona $5 \mathrm{mg} \cdot \mathrm{kg} \cdot$ dosis im. sólo mientras persistiese su gravedad. El alia se dio con un puntaje de Tal de 2 o menos.

El estudio extadístico se efectuó mediante las dócimas t de Student. Mann Withney, ANOVA y Kruskal Wallis según la cantidad de grupos a comparar y el cumplimiento de supuestos estadisticos. Los cálculos estadísticos se hicieron mediante el programa EPI INFO versión .5.

\section{Resultados}

La edad de los 25 pacientes de cada grupo (I: 7,52 \pm 5,76; $\mathrm{NaCl}: 6,84 \pm 4,34 ; \mathrm{FI}: 6,96 \pm$ 4,$22 ; S: 7,52 \pm 4,47$ ) se distribuyó uniformemente, predominando en todos ellos los lactantes menores de un año. Los pacientes eran de sexo masculino en I: $14 / 25$ casos, $\mathrm{NaCl}$ : 11/25. FI: $16 / 25$ y S: $15 / 25$.

El puntaje de Tal al ingreso fue semejante en todos los grupos, produciéndose una disminución estadísticamente significativa en favor del grupo que recibe fenoterol-ipratropio desde el día 1 de tratamiento, la que se acentuó a contar del día 2 (figura 1). En tabla 2 se detallan las diferencias estadísticas obtenidas, no especificándose las de los días siguientes de tratamiento por ser muy pequeño el número de pacientes que por grupos se mantenía en el estudio.

El día 2 de tratamiento fueron dados de alta dos pacientes, ambos del grupo FI y al día 4 de tratamiento se había dado de alta a 4 (16\%) pacientes tratados con I, $3(12 \%)$ con $\mathrm{NaCl}, 19$ (76\%) pacientes con FI y $5(20 \%)$ de pacientes con $\mathrm{S}$. Encontrándose una diferencia estadísticamente significativa $(\mathrm{p}<0,001)$ al comparar los días de estada de los niños tratados con FI ante los que recibieron $I, \mathrm{NaCl}$ y $S$; no así al comparar entre sí al resto de los grupos (I vs $\mathrm{NaCl} p=0,055$, I vs $\mathrm{S} p=0,136$ y $\mathrm{S}$ vs $\mathrm{NaCl}$ $\mathrm{p}=0,733$ ).

Sólo a un paciente $(4 \%)$ tratado con FI fue necesario agregar salbutamol por inhalador el día 2 de tratamiento, mientras que, en ese momento del estudio, $3(12 \%)$ pacientes con I, 10 (40\%) con $\mathrm{NaCl}$ y $6(24 \%)$ con $\mathrm{S}$ recibían olro broncodilatador. El grupo que recibió FI fue el que en menos pacientes requirió usar otro broncodilatador desde el día 1 de tratamiento $(\mathrm{p}<$ $0,01)$. Por el contrarjo, los niños que recibieron solo $\mathrm{NaCl}$ fueron los que más tratamiento adicional demandaron $(\mathrm{p}<0,05)$ desde el día 2 de tratamiento (figura 3 ). El uso de corricoides parenterales fue necesario en 3 pacientes ( $12 \%$ ) que recibian I, 4 (l6\%) que recibían $\mathrm{NaCl}, 3$ (12\%) con Fl y $5(20 \%)$ con S. Sin embargo, estas diferencias no son estadísticamente significativas.

Tabla 1

Puntaje de Tal moditicado

\begin{tabular}{|c|c|c|c|c|c|}
\hline Puntaje & $<6 \mathrm{~m}$ & $>6 \mathrm{~m}$ & $\begin{array}{c}\text { Retracción } \\
\text { partes blandas }\end{array}$ & Sibilancias & Clanosis \\
\hline 0 & $<40$ & $<30$ & No & No & No \\
\hline $\mathbf{J}$ & $41-55$ & $31-45$ & + & Espiración & Peribucal al llorar \\
\hline 2 & $56-70$ & $46-60$ & +t & Insp y esp & Peribueal en reposo \\
\hline 3 & $>70$ & $>60$ & +++ & $\begin{array}{l}\text { Audible a } \\
\text { distancia }\end{array}$ & Generalizada \\
\hline
\end{tabular}

Esp: espiratorias Insp: inspiratorias

$0-4$ puntos = obstrucción brouquial leve

$5-8$ puntos $=$ obstrucción bronquial moderada

$9-12$ puntos = obstrucción bronquial grave 
TAL

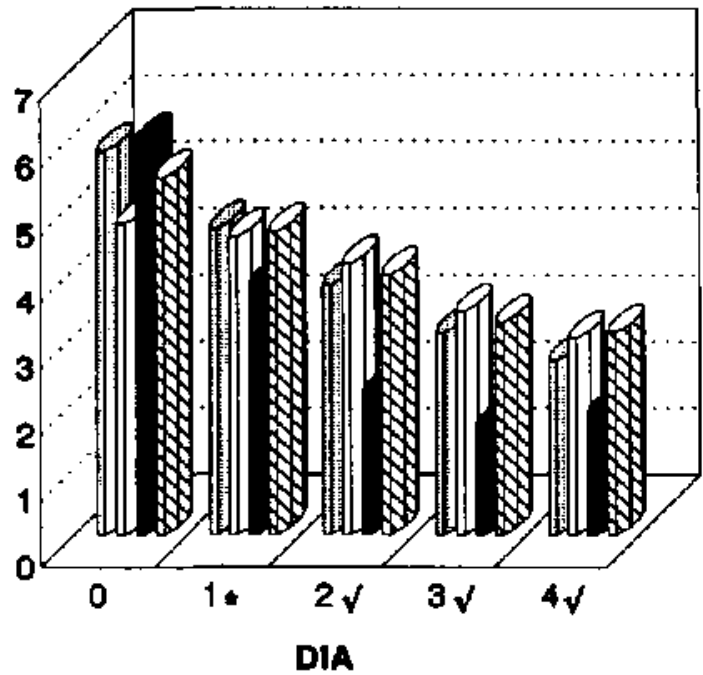

$-p<0,05$ ( GRUPO 3 )

$\sqrt{p}<0,01$ ( GRUPO 3 )

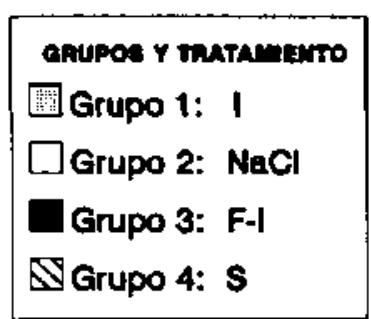

Figura 1: Promedius dél puntaje de Tal en los primeros cuatro dias de tratamiento ex lactantes con sindrome bronquial obstructivo tratados con ipratropio (I). $\mathrm{NaCl}$, fenoterol ipratropio (FI) y salbutamol (S).

Tabla 2

Diferencias estadísticas (p) en puntaje de Tal en Ios tres primeros días de tratamiento con ipratropio (grupo 1), $\mathrm{NaCl}$ (grupo 2), fenoterol ipratropio (grupo 3) y salbutamol (grupo 4)

\begin{tabular}{llll}
\hline Grupos & $\begin{array}{c}\text { Día 1 } \\
\text { (p) }\end{array}$ & $\begin{array}{c}\text { p } \\
\text { Día 2 } \\
\text { (p) }\end{array}$ & $\begin{array}{c}\text { Dia 3 } \\
\text { (p) }\end{array}$ \\
\hline 1 vs 2 & 0,711 & 0,322 & 0,393 \\
I v5 3 & $0,018^{*}$ & $0,000^{*}$ & $0,000^{*}$ \\
I v5 4 & 0,825 & 0.817 & 0,777 \\
2 v5 3 & $0,029^{*}$ & $0,000^{*}$ & $0,000^{*}$ \\
2 vs 4 & 0,927 & 0,423 & 0,537 \\
3 v5 4 & $0,014^{*}$ & $0,000^{*}$ & $0,000^{*}$ \\
\hline
\end{tabular}

* Estadísticamente significativo
En ninguno de los grupos se detectaron efectos secundarios indeseables, ya fuera por la nebulización o el medicamento utilizado. Al contrario, la frecuencia cardíaca elevada al ingreso del paciente disminuye en la medida que cedia el cuadro bronquial obstructivo independiente de él o los medicamentos utilizados.

\section{Comentario}

Aun no se han desarrollado criterios comunes para el tratamiento con broncodilatadores de lactantes con crisis bronquial obstructiva, pese a más de 15 años de experiencia ${ }^{8}$. Hay, sin embargo, consenso en que, si bien es cierto no to-

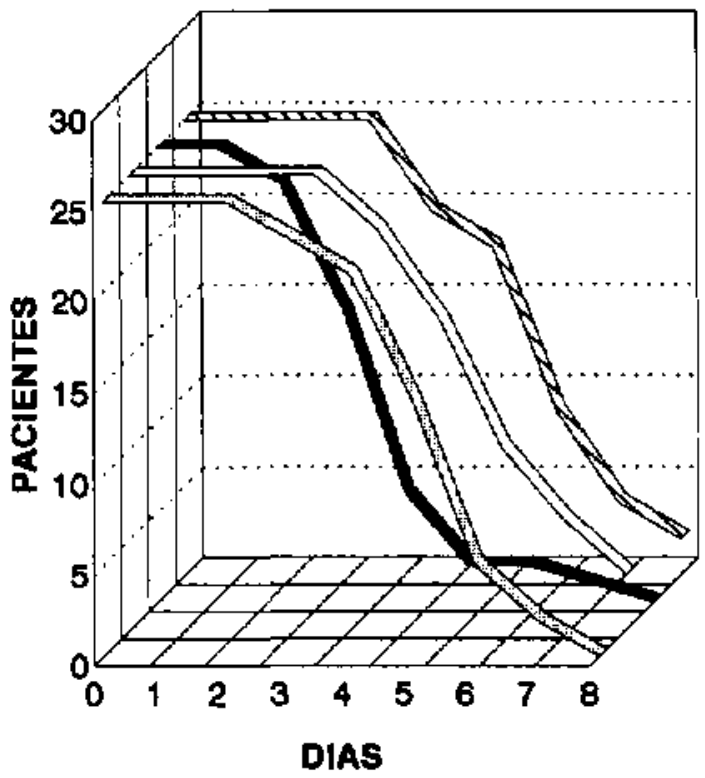

$P<0,001$ (GRUPO 3)

$$
\begin{aligned}
& \text { DUE HOEPITALIZACIOM (x) } \\
& \text { G } 1: 4,64 \pm 1,29 \\
& \square \text { G } 2 \text { : } 5,44 \pm 1,61 \\
& \text { G } 3: 3,0 B \pm 1,28 \\
& \mathbb{S G}_{4}: 5,28 \pm 1,69
\end{aligned}
$$

Figura 2: Duración de la hospitalización en laciantes con sindrome bronquial obstcuctivo tratados con ipratropio (G1). $\mathrm{NaCl}$ (G2), fenoterol ipratropio (G3) y salbutamol (G4). 


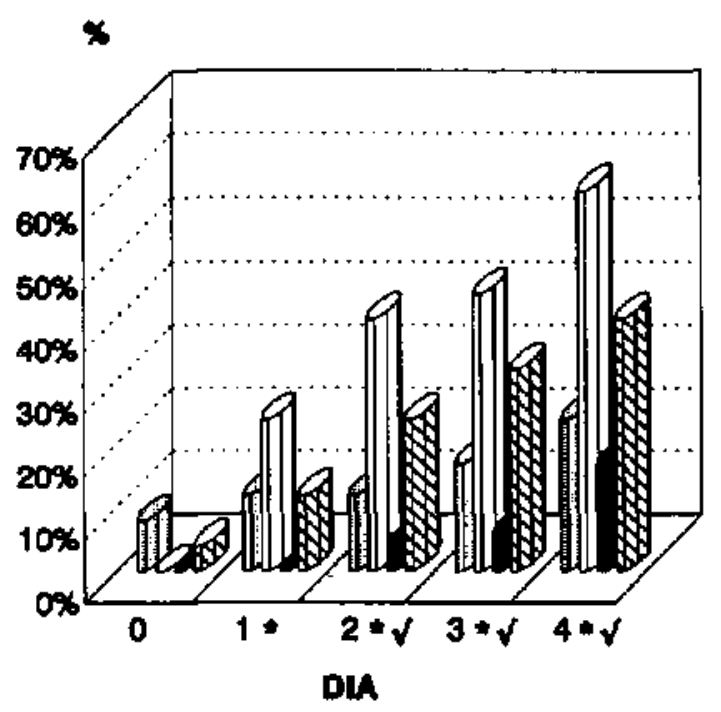

* $p<0,01$ ( GPUPO 3 )

$\checkmark p<0,06$ ( GRUPO 2 )

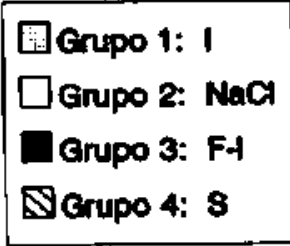

Figura 3: Proporciones de lactantes con sindrome bronquial obstructivo ranejados con ipralropio (I). $\mathrm{NaCl}$, fenoterol ipsatropio ( $\mathbf{F l}$ ) y salbutamol $(S)$, que requirieron otro broncodilatador.

dos los lactantes responderán a los broncodilatadores, un número importante de ellos se beneficiará con su empleo, por 10 que deben ser usados, máxime si una proporción significativa de los afectados sufre asma bronquial.

Previamente se había demosirado' que los lactantes tratados con FI evolucionan más favorablemente que los que reciben $\mathrm{NaCl} 0,9 \%$. Ahora interesaba conocer si la respuesta era mejor con un beta 2 adrenérgico, un anticolinérgico a la mezcla de ambos. La respuesta según puntaje clínico de Tal, los días de estada o la necesidad de terapia adicional con otro broncodilatador, fue francamente mejor en los niños que recibieron la mezcla FI. La excelente respuesta a los beta 2 adrenérgico de los niños mayores de dos años no se consigue en los lactantes, siendo aún discordantes los diferentes reportes sobre la materia ${ }^{9 \cdot 1]}$. Por ocra parte, consi- derando que el tono bronquial del músculo liso de la vía respiratoria se debe en gran parte a impulsos parasimpáticos colinérgicos ${ }^{12}, 13$, se ha estimulado el uso de agentes anticolinérgicos individuales o combinados con beta 2 adrenérgicos, sin resultados concluyentes ${ }^{5.14 .15}$.

En nuestra experiencia la combinación de fenoterol e ipratropio tiene una indicación preferencial en cl tratamiento de lactantes con síndrome bronquial obstructivo. Salbutamol e ipratropio mostraron resultados similares y, aunque éstos fueron mejores que con $\mathrm{NaCl}$, las diferencias no son estadísticamente significativas. Puesto que por motivos éticos el protocolo dis. puso que a los pacientes sin evolución favorable al cabo de 24 horas se les tratase con un broncodilatador claramente identificado por el médico tratante, se explica que $64 \%$ de los pacientes manejados con $\mathrm{NaCl}$ recibiesen tratamiento con salbutamol en inhalador al día 4 de ingresar.

De estos resultados es posible deducir que un grupo importante de lactantes obstruidos, independicnte de la edad, responderá al uso de broncodilatadores, siendo ésta una medida terapéutica fundamental. De los medicamentos disponibles para estos efectos en niños de esta edad, se debería optat en primer lugar por la mezcla de fenoterol e ipratropio en nebulización, quedando como alternativa de segunda línea el uso de ipratropio o salbutamol si no contamos con la presentación farmacéutica indicada.

\section{Resumen}

Se evalúa la respuesta clínica a diferentes broncodilatadores en 100 lactantes con síndrome bronquial obstructivo que, por la intensidad de éste requerían hospitalización, mediante un estudio prospectivo doble ciego, en el que se asignaron 25 niños menores de lactantes por grupo. Los niños del grupo I recibieron ipratropio (I), los del grupo II cloruro de sodio $9 \%$. $(\mathrm{NaCl})$ : en el grupo III se emplé fenoterolipratropio (FI) y en el grupo IV salbutamol (S), en todos los casos por nebulización. La calificación de Tal era similar al ingreso en los 4 grupos (día 0). Al día 1 de tratamiento era significativamente menor $(\mathrm{p}<0,05)$ en FI, como también el día 2 (p < 0,01): (promedios día 1: 4,6 (I); 4,5 (NaCl); 3.8 (FI); 4,6 (S); día 2: 3,8 (I) 4,1 (NaCl); 2,1 (FI); 3,9 (S); dia $3: 3,0$ (I); 3,4 
(NaCl); I,6 (FI); 3,2 (S). El promedio de días estada fue I: $4,64 \pm 1,29 ; \mathrm{NaCl}: 5,44 \pm 1,61$; FI: $3,08 \pm 1,28 ;$ S: $5,28 \pm 1,69 ; p<0,001$ a favor de Fl. Los niños tratados con $\mathrm{NaCl}$ requirieron con mayor frecuencia terapia adicional con broncodilatadores $(p<0,05)$ en claro contraste con los manejados con $\mathrm{Fl}$, que requirjeron menos broncedilatadores adicionales que el resto $(\rho<$ 0,01 ) desde el día l del ingreso. No se registraron efectos secundarios indeseables con los djferentes regímenes aplicados.

(Palabras claves: lactante, sindrome bronquial obstructivo, fenoterol-ipratropium, salbutamol, ipratropium.)

\section{Referencias}

1. Calwo M. Grob K. Herrmann M. Marín F: Nebulizaciones de fenotecol-ipratropio en lactantes con síndrome bronquial obstructivo. Rev Chil Pediatr 1990; 61: 193-197.

2. Bentur L. Canny G. Shields M. et al: Controlled trial of nebulized albuterol in children younger than 2 years of age with acute asthma. Pediatrics 1992;89; 133-137.

3. Taf A, Le'y $N$. Bearmun f: Methylprednisolone therapy for acute asthma in infants and toddlers: a controlled clinical trial. Pediatrics 1990: 86: 350-356.

4. Wand $E$, Milner $R$, Allen $U$, Maj $H$ : Bronchodilators for treatment of mild bronchiolitis: a factorial randornised trial. Arch Dis Chil 1992; 67: $389-293$.
5. Schuh S, Johnson D, Canny G, et al: Efficacy of adding nebulized ipratropium bromide to nebulized albuterol therapy in acute bronchiolitis. Pediatrics 1992; 90: 920-923.

6. Ho L, Collis G, Landan L, Le-Sovef P: Effect of salbutanol on oxygen saturation in bronchiolitis. Atch Dis Child 1991: 66: 106!-1064.

7. Tal A, Bavilski Ch, Yohai D. et al: Dexamethasone and salbutamol in the treatment of acute wheezing in infants. Pediatries 1983; 71: 13-18.

8. Lenney $N$. Milner $A$ : At what age to bronchodilator drugs work?. Arch Dis Chil 1978; 53:502-535.

9. Schuth S. Canny G. Reisman J, et al: Nebulized alhuterol in acute bronchiolitis. J Pediatr [990; 117 : 633-637.

10. Klassen T, Rowe P. Sutciffe T. Ropp L, Mc Dowell I. $L i M$ : Randomized trial of salbutamol in acute bronchiolitis. J Pediatr 1991; $118: 807-811$.

11. Lin $Y^{\prime}$, Asieh $K$, Chen $W_{1}$ Wit $K$ : Clinical trial of corticosteroid and $B 2$ bronchodilator in acute wheezing infants. Acta paediatr Scan 1991; 32; 333340 .

12. Gross N: Drug therapy: ipratropium bromide. N Engl J Med 1988; 319: 486-494.

13. Widdicumbe $J$ : Neurohormonal mechanisms in obstructive aitways disease. En Gross N, ed. Anticholinergic therapy in obstructive airways disease, Londres. Franklin Scientific Publications, 1993; 33-44.

14. Prenduille A, Green $S$, Silverman $M$; Ipratropium bromide and airways function in wheezy infants. Ach Dis Chil 1987;62: 397-420.

15. Henry $R$, Milner A, Stokes $G$ : Ineffectiveness of ipratropium bromide in acule bronchiolitis. Arch Dis Child 1983; $58: 925-926$. 